\title{
Oxidative stress markers predict early left ventricular systolic dysfunction after acute myocardial infarction treated with primary percutaneous coronary intervention
}

\author{
Dubravka Rajic ${ }^{1, A-D}$, Ivica Jeremic 2, 3, A-D , Sanja Stankovic ${ }^{4, B, C, E}$, Olivera Djuric ${ }^{3,5, C, D}$, Tatjana Zivanovic-Radnic 2, B, \\ Igor Mrdovic 1,3, C, E, F, Predrag Mitrovic ${ }^{1,3, A}$, Dragan Matic ${ }^{1,3, B}$, Zorana Vasiljevic 3, E, Mihailo Matic 3, A, E, Milika Asanin 1, 3, C, E, F \\ ${ }^{1}$ Cardiology Clinic, Clinical Centre of Serbia, Belgrade, Serbia \\ ${ }^{2}$ Institute of Rheumatology, Belgrade, Serbia \\ ${ }^{3}$ School of Medicine, University of Belgrade, Serbia \\ ${ }^{4}$ Center for Medical Biochemistry, Clinical Center of Serbia, Belgrade, Serbia \\ ${ }^{5}$ Institute of Epidemiology, Belgrade, Serbia
}

A - research concept and design; $\mathrm{B}$ - collection and/or assembly of data; $\mathrm{C}$ - data analysis and interpretation; $D$ - writing the article; $E$ - critical revision of the article; $F$ - final approval of the article

Address for correspondence

Ivica Jeremic

E-mail: ivicaje@yahoo.com

Funding sources

This study was supported by the Serbian Ministry of Education, Science and Technological Development (http://www.mpn.gov.rs/) through the Research Project No. III 41025. The funders had no role in study design, data collection and analysis, decision to publish, or preparation of the anuscript.

Conflict of interest

None declared

Received on March 25, 2016

Reviewed on June 17, 2016

Accepted on August 3, 2016

DOI

10.17219/acem/64464

Copyright

Copyright by Author(s)

This is an article distributed under the terms of the

Creative Commons Attribution Non-Commercial License

(http://creativecommons.org/licenses/by-nc-nd/4.0/)

\begin{abstract}
Background. Despite successful primary percutaneous coronary intervention (PCI) after ST-segment elevation myocardial infarction (STEMI), some patients develop left ventricular systolic dysfunction (LVSD) and acute heart failure (HF). Identifying patients with an increased risk of developing LVSD by means of biomarkers may help select patients requiring more aggressive therapy.

Objectives. The aim of this study was to evaluate the relationship between the levels of oxidative stress markers and development of LVSD and acute HF early after STEMI.

Material and methods. The study enrolled 148 patients with the first STEMI, who were treated by primary $\mathrm{PCl}<12 \mathrm{~h}$ from the onset of symptoms. We assessed the impact of different biomarkers for developing LVSD and acute HF (Killip $\geq 2$ ) including: markers of necrosis - peak creatine kinase (CK), markers of myocardial stretch - B-type natriuretic peptide (BNP), inflammatory markers - C-reactive protein (CRP), leucocyte and neutrophil count, as well as oxidative stress markers - total thiol groups, catalase, superoxide dismutase (SOD) and glutathione reductase (GR).

Results. In multivariate analysis, thiol groups, peak CK, anterior wall infarction, and age were predictors of LVEF $\leq 40 \%$. Out of 16 variables significantly associated with the Killip $\geq 2$ in univariate logistic regression analysis, 5 appeared to be independently associated with acute HF in multivariate analysis: catalase, BNP, leucocytes, neutrophil count, and size of left atrium.

Conclusions. In this study, we have shown for the first time that thiol groups and catalase are independent predictors of STEMI complication - LVSD and acute HF, respectively. Beside routine used biomarkers of necrosis and myocardial stretch, thiol groups and catalase may provide additional information regarding the risk stratification.
\end{abstract}

Key words: oxidative stress, heart failure, acute myocardial infarction, percutaneous coronary intervention, left ventricular systolic dysfunction 


\section{Introduction}

Primary percutaneous coronary intervention (PCI) is a first choice reperfusion therapy for ST-segment elevation myocardial infarction (STEMI). Primary PCI, in most cases, provides early and complete restoration of blood flow in the infarct related artery (IRA) and improves myocardial viability, ventricular function and patient survival. ${ }^{1}$ Despite primary PCI, some patients develop left ventricular systolic dysfunction (LVSD) and acute heart failure (HF), which have an adverse impact on short and long-term outcomes. ${ }^{2}$ The sudden reperfusion of occluded artery leads to injuries mainly induced by oxidative and inflammatory mechanisms. Restored oxygen supply increases free radical production in the electron transport chain on the inner mitochondrial membrane, while inflammatory cells, especially neutrophils, release free oxygen species and cytokines with negative inotropic effects and further aggravate myocardial dysfunction and contribute to HF. ${ }^{3}$ Early identification of patients at an increased risk of developing left ventricular (LV) dysfunction after acute myocardial infarction (AMI) could help in the selection of patients requiring more aggressive therapy. The transthoracic echocardiographic examination remains the most often used method for non-invasive detection of LV dysfunction and risk stratification after AMI. Ejection fraction (EF) or other closely related parameters are strong predictors of the risk for future events. ${ }^{4}$

Besides a physical examination and an ultrasound, a laboratory evaluation has an essential role. Biomarkers are indispensable tools to present a diagnosis and prognosis in acute coronary events, and some of them are now introduced into the current guidelines for HF. ${ }^{5}$

Most data on biomarkers has been derived from patients with chronic HF, but risk stratification in patients with acute HF remains a challenge. In the development of LVSD and $\mathrm{HF}$ after AMI participate different pathophysiological processes, which are reflected by different biomarkers. ${ }^{6}$ Apart from the commonly used B-type natriuretic peptide (BNP) and N-terminal pro-brain natriuretic peptide (NT-proBNP), risk stratification may be refined by the use of new biomarkers. ${ }^{7}$ Markers of oxidative stress are also widely studied in chronic HF, but their role in the prediction of early systolic dysfunction after STEMI is largely unknown. We focused on oxidative stress parameters that are cheap, easy to perform and widely available. Thiol groups are found in many proteins and have an essential role for their function. Thiol/disulphide homeostasis is disrupted in AMI and could serve as an additional marker for diagnosis. ${ }^{8}$ Catalase, another very often used marker of oxidative stress, was found to be increased in an end-stage failing heart, while higher catalase activity was observed in AMI as well in subjects with high cardiovascular risk. ${ }^{9,10}$ Considering the important role of oxidative stress on myocardial injury during the reperfusion, we postulated that lower antioxidant status early after AMI will predict development of low ejection fraction.

In this paper, we described the role of markers of oxidative stress in the prediction of left ventricular systolic dysfunction and acute heart failure early after AMI.

\section{Patients and methods}

\section{Patients}

The study enrolled 148 patients aged 18 years or more, with the first STEMI, who were treated by primary PCI $<12$ h from the symptoms onset. Patients were admitted to the Coronary Care Unit of Clinical Centre of Serbia, from October 2013 to December 2014. All patients signed consent forms, whilst the study was approved by the Ethical Committee of Clinical Centre of Serbia. The primary endpoint of this study was to establish if oxidative stress markers were predictors of early LVSD in patients with STEMI. The role of oxidative stress markers in the prediction of acute HF was a secondary endpoint.

The STEMI was defined according to European Society of Cardiology guidelines. ${ }^{11}$ A standard 12-lead electrocardiography (ECG) was performed on admission and $60 \mathrm{~min}$. after revascularization with PCI. Reduction of $\geq 50 \%$ in ST-segment elevation after PCI was considered significant resolution of ST-segment elevation (STR). ${ }^{12}$ The exclusion criteria were: the presence of any contraindication to dual antiplatelet therapy and contrast agents, inability or refusal to provide written informed consent, prior coronary revascularisation, prior history of heart failure, permanent atrial fibrillation, left bundle branch block, hemodynamically severe valvular heart disease, primary cardiomyopathies, chronic pulmonary disease, implantable pacemaker, acute or chronic inflammation, history of autoimmune disease, history of febrile disorders and malignancy.

Coronary angiography and primary PCI were performed using the standard technique. Patients were treated on admission and after PCI according to current guidelines. ${ }^{11}$ The angiogram of all patients was graded according to the number of diseased coronary arteries. The coronary flow in the IRA was graded according to the classification used in the thrombolysis in myocardial infarction (TIMI) trial. Successful reperfusion was defined as the establishment of TIMI grade 3 flow in the IRA. ${ }^{13}$ Time from symptoms onset to the treatment was defined as the time from the symptom onset to the first balloon inflation.

All patients underwent astandard transthoracic echocardiography on the $4^{\text {th }}$ or $5^{\text {th }}$ day of admission. The size of the heart chambers, left ventricular ejection fraction and wall motion score index (WMSI) were assessed. LVEF was calculated by modified biplane Simpson's method and defined as EF $\leq 40 \%$. 5,14 According to the values of LVEF, patients were divided into groups with LVSD (the group with LVEF $\leq 40 \%$ ) and without LVSD (the group 
with LVEF > 40\%). WMSI was calculated according to the 17-segment model division of the LV and assigned a score of $1,2,3$, and 4 points for normokinesia, hypokinesia, akinesia, and dyskinesia, respectively. ${ }^{14}$ Significant regional LVSD was considered WMSI $\geq 1.5$. Patients who developed HF during hospitalization were categorized according to the Killip status of I-IV: class I - no rales and no third heart sound; class II - pulmonary congestion with rales up to $50 \%$ of the lung fields, sinus tachycardia or third heart sound; class III - pulmonary edema with rales over $50 \%$ of the lung fields; and class IV - included cardiogenic shock. ${ }^{11}$ Killip of class $\geq 2$ was considered as acute HF.

\section{Blood sampling and biochemical analysis}

Blood samples were collected after admission and before PCI. Plasma and serum were derived, aliquoted and immediately frozen at $-80^{\circ} \mathrm{C}$ until analysis. Routine laboratory analyses were determined from separated samples obtained at the same time. Creatine kinase (CK), B-type natriuretic peptide (BNP), C-reactive protein (CRP), complete and differential blood count were determined according to standard laboratory procedures. Glutathione reductase (GR) was determined by using commercially available kits according to manufacturer instructions (Randox Laboratories Ltd., Crumlin, UK). Total thiol groups were determined by Ellman's reagent (5,5-dithiobis-(2-nitrobenzoic acid)-DTNB) as previously described. ${ }^{15}$ Catalase was determined exactly as previously described. ${ }^{16}$ Superoxide dismutase (SOD) was measured according to method based on autoxidation of adrenaline. ${ }^{17}$

\section{Statistical analysis}

Distribution of the continuous variables was assessed using the Kolmogorov-Smirnov test. Depending on the normality, continuous data was presented as mean \pm SD or median (interquartile range - IQR). Categorical data is summarized as numbers (percentages). Comparison of continuous features of patients with and without HF was done using the Student's t-test or Mann-Whitney U test. Pearson's $X^{2}$ test or the Fisher's exact test were used for comparing categorical characteristics between the 2 groups. Spearman's correlation were used to estimate the association of LVEF with biochemical and echocardiographic parameters. In order to estimate which of the biochemical, clinical and echocardiographic parameters from a baseline were independently associated with LVEF $\leq 40 \%$ and clinically apparent $\mathrm{HF}$ (Killip $\geq 2$ ), univariate and multivariate logistic regression analyses were done. Variables that were significantly associated with HF in univariate logistic regression at the significance level $<0.1$ were entered into the multivariate logistic regression model (Hosmer-Lemeshow method). ${ }^{18}$ Odds ratios (OR) with 95\% confidence intervals were computed and Pearson goodness of fit test was performed to assess the overall fitness of the models. All statistical tests were two-sided and were performed at the $5 \%$ significance level. The statistical analysis was performed using IBM SPSS Statistics v. 20.0 software (IBM Corp., Armonk, USA).

\section{Results}

The study included 148 patients (111 men and 37 women) with a mean age of $58.7 \pm 11.7$ years. Baseline characteristics of patients are shown in Table 1. The patients with LVSD were more frequently male, with an anterior wall infarction, heart rhythm disorders, acute HF, and significantly lower STR. There were no significant differences between the 2 groups with regard to the risk factors and renal function.

Left ventricular end-diastolic diameter (LVEDD), left ventricular end-systolic diameter (LVESD) and the left atrial (LA) size were significantly higher in the group with $\mathrm{LVEF} \leq 40 \%$. Additionally, WMSI $\geq 1.5$ was more prevalent in this group of patients (61 (88.4\%) vs $2(2.5 \%)$; $<<0.001)$ ). Angiographic data revealed that TIMI flow grade after the primary PCI, presence of multi-vessel coronary disease and the number of the implanted stents did not differ between the 2 groups; however, there was a significantly higher prevalence of left anterior descending (LAD) artery as the culprit artery in group with LVEF $\leq 40 \%$. During hospitalization, 4 patients with LVSD died, while none of the patients died in the group without LVSD $(\mathrm{p}<0.001)$.

Thiol groups, as a marker of oxidative stress, were significantly lower in the group with LVEF $\leq 40 \%(\mathrm{p}=0.034)$, while the peak CK, a marker of myocardial necrosis, was significantly higher in the group with reduced LVEF than in patients with LVEF $>40 \%$ (Table 2). The inflammatory parameters, CRP, leukocytes and neutrophils were significantly higher in the group with LVEF $\leq 40 \%$ compared with patients who had LVEF $>40 \%(\mathrm{p}=0.006),(\mathrm{p}<0.001)$ and $(\mathrm{p}=0.011)$, respectively. The same results were observed for markers of myocardial stretch. Plasma levels of BNP were higher in the group of patients with LVSD than patients without LVSD ( $\mathrm{p}<0.001)$.

Regarding the acute HF during hospitalization, patients who developed Killip $\geq 2$ had significantly lower concentration of thiol groups but higher concentrations of peak CK, CRP, leukocyte and neutrophil count compared to patients with Killip $<2$ (Table 3).

Spearman's correlation showed significant association between LVEF and thiol groups $(\mathrm{r}=0.187 ; \mathrm{p}=0.023)$, and negative correlation with peak $\mathrm{CK}(\mathrm{r}=-0.505$; $\mathrm{p}<0.001)$, plasma BNP levels $(r=-0.326$; $\mathrm{p}<0.001)$, leukocytes $(\mathrm{r}=-0.384 ; \mathrm{p}<0.001)$, neutrophils $(\mathrm{r}=-0.223 ; \mathrm{p}=0.006)$ and CRP $(\mathrm{r}=-0.261 ; \mathrm{p}=0.001)$. Considering echocardiographic features, LVEF showed negative correlation with WMSI ( $\mathrm{r}=-0.924$; $\mathrm{p}<0.001)$, LVEDD $(\mathrm{r}=-0.480$; $\mathrm{p}<0.001)$ and LVESD $(\mathrm{r}=-0.480 ; \mathrm{p}<0.001)$. 
Table 1. Baseline characteristics of patients with and without left ventricular systolic dysfunction

\begin{tabular}{|c|c|c|c|}
\hline Variable & $\begin{array}{l}\text { LVEF } \leq 40 \% \\
\quad(n=69)\end{array}$ & $\begin{array}{l}\text { LVEF }>40 \% \\
\quad(n=79)\end{array}$ & $p$-value \\
\hline \multicolumn{4}{|c|}{ Baseline characteristics } \\
\hline Age $[\text { years] }]^{+}$ & $58.88 \pm 12.43$ & $58.51 \pm 11.42$ & 0.848 \\
\hline Male gender, n (\%) & $59(85.5)$ & $52(65.8)$ & 0.006 \\
\hline $\mathrm{BMI}\left[\mathrm{kg} / \mathrm{m}^{2}\right]^{\dagger}$ & $28.04 \pm 4.06$ & $26.83 \pm 4.14$ & 0.075 \\
\hline Smokers, n (\%) & $45(65.2)$ & $48(60.8)$ & 0.576 \\
\hline Hypertension, n (\%) & $38(55.1)$ & $55(69.6)$ & 0.068 \\
\hline Diabetes, n (\%) & $11(15.9)$ & $20(25.3)$ & 0.162 \\
\hline Hyperlipidemia, n (\%) & $44(63.8)$ & $49(62.0)$ & 0.827 \\
\hline Family history, n (\%) & $21(30.4)$ & $30(38.0)$ & 0.336 \\
\hline eGFR < 60 mL/min, n (\%) & $9(13.0)$ & $5(6.3)$ & 0.164 \\
\hline \multicolumn{4}{|c|}{ Clinical features } \\
\hline $\begin{array}{l}\text { Time from onset of chest } \\
\text { pain to } \mathrm{PCl}[\mathrm{min}]^{*}\end{array}$ & $300(345.0)$ & $240(240)$ & 0.057 \\
\hline Anterior wall infarction, n (\%) & $62(89.9)$ & 25 (31.6) & $<0.001$ \\
\hline Killip class $\geq 2$, n (\%) & $13(18.8)$ & $3(3.8)$ & $<0.001$ \\
\hline \multicolumn{4}{|c|}{ Echocardiography } \\
\hline $\operatorname{LVESD}[\mathrm{cm}]^{\dagger}$ & $4.37 \pm 0.58$ & $3.44 \pm 0.41$ & $<0.001$ \\
\hline 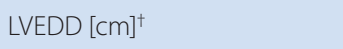 & $5.76 \pm 0.62$ & $5.24 \pm 0.45$ & $<0.001$ \\
\hline $\mathrm{LA}[\mathrm{cm}]^{\dagger}$ & $3.89 \pm 0.54$ & $3.68 \pm 0.38$ & 0.009 \\
\hline WMSI $\geq 1.5, \mathrm{n}(\%)$ & $61(88.4)$ & $2(2.5)$ & $<0.001$ \\
\hline \multicolumn{4}{|c|}{ Electrocardiography } \\
\hline STR, n (\%) & $55(79.7)$ & $77(97.5)$ & 0.001 \\
\hline Ventricular arrhythmias, n (\%) & $11(15.9)$ & $3(3.8)$ & 0.001 \\
\hline \multicolumn{4}{|c|}{ Angiographic findings } \\
\hline Multi-vessel disease, n (\%) & $34(49.3)$ & $41(51.9)$ & 0.750 \\
\hline \multicolumn{4}{|l|}{ IRA, n (\%) } \\
\hline LM & $1(1.4)$ & $1(1.3)$ & $<0.001$ \\
\hline$L A D$ & $61(88.4)$ & $21(26.6)$ & \\
\hline LCx & $4(5.8)$ & $11(13.9)$ & \\
\hline RCA & $3(4.3)$ & $46(58.2)$ & \\
\hline Number of stents implanted ${ }^{*}$ & $1(0)$ & $1(1)$ & 0.795 \\
\hline \multicolumn{4}{|l|}{ Final TIMI flow, n (\%) } \\
\hline TIMI flow 0 & 0 & 0 & 0.598 \\
\hline TIMI flow 1 & $0(0.0)$ & $0(0.0)$ & \\
\hline TIMI flow 2 & $2(2.9)$ & $1(1.3)$ & \\
\hline TIMI flow 3 & $67(97.1)$ & $78(98.7)$ & \\
\hline \multicolumn{4}{|c|}{ Therapy, n (\%) } \\
\hline ACE inhibitors & $56(81.2)$ & 77 (97.4) & 0.51 \\
\hline Beta blockers & $62(89.9)$ & $70(88.6)$ & 0.114 \\
\hline Diuretics & $25(36.2)$ & $4(5.1)$ & $<0.001$ \\
\hline Digitalis & $8(11.6)$ & $5(6.3)$ & 0.068 \\
\hline Antiarrhythmics & $17(24.6)$ & $3(3.8)$ & $<0.001$ \\
\hline In-hospital mortality, n (\%) & $4(5.8)$ & $0(0)$ & $<0.001$ \\
\hline
\end{tabular}

Table 2. Biomarkers of patients with and without left ventricular systolic dysfunction

\begin{tabular}{|c|c|c|c|}
\hline Variable & $\begin{array}{l}\text { LVEF } \leq 40 \% \\
\quad(n=69)\end{array}$ & $\begin{array}{l}\text { LVEF }>40 \% \\
\quad(n=79)\end{array}$ & $p$-value \\
\hline \multicolumn{4}{|c|}{ Oxidative stress } \\
\hline Thiol groups $[\mu \mathrm{M}]^{*}$ & $39.4(14.5)$ & $44.6(22.0)$ & 0.034 \\
\hline Catalase [U/mg Prot $]^{¥}$ & $98.20(7.20)$ & $96.1(10.70)$ & 0.161 \\
\hline $\mathrm{GR}[\mathrm{U} / \mathrm{mL}]^{+}$ & $8.94 \pm 8.88$ & $8.00 \pm 7.57$ & 0.536 \\
\hline $\mathrm{SOD}[\mathrm{U} / \mathrm{mL}]^{\dagger}$ & $2.91 \pm 1.19$ & $3.16 \pm 0.89$ & 0.141 \\
\hline \multicolumn{4}{|c|}{ Necrosis } \\
\hline Peak CK $[U / L]^{*}$ & $3153(3867.5)$ & $1162(1432)$ & $<0.001$ \\
\hline \multicolumn{4}{|c|}{ Strech } \\
\hline $\mathrm{BNP}[\mathrm{pg} / \mathrm{mL}]^{*}$ & $49(156.8)$ & $14.9(53.8)$ & $<0.001$ \\
\hline \multicolumn{4}{|c|}{ Inflammation } \\
\hline Sedimentation $[\mathrm{mm} / \mathrm{h}]^{*}$ & $18.0(22.0)$ & 19 (19.75) & 0.886 \\
\hline C-reactive protein $[\mathrm{mg} / \mathrm{L}]^{*}$ & $12.1(33.0)$ & $8.4(8.4)$ & 0.006 \\
\hline Leukocyte count $\left[\times 10^{9} / \mathrm{L}^{+}\right.$ & $12.58 \pm 4.03$ & $10.23 \pm 2.45$ & $<0.001$ \\
\hline Neutrophil count $\left[\times 10^{9} / \mathrm{L}\right]^{*}$ & $83.3(10.9)$ & $79(11.3)$ & 0.011 \\
\hline Fibrinogen $[\mathrm{g} / \mathrm{L}]^{*}$ & $2.0(1.36)$ & $2(0.0)$ & 0.078 \\
\hline
\end{tabular}

${ }^{\dagger}$ values are mean $\pm \mathrm{SD} ;{ }^{*}$ values are median $(\mathrm{IQR}) ; \mathrm{GR}$ - glutathione reductase; $\mathrm{SOD}$ - superoxide dismutase; $\mathrm{CK}$ - creatine kinase BNP - B-type natriuretic peptide.

Table 3. Biomarkers of patients with and without acute heart failure

\begin{tabular}{|c|c|c|c|}
\hline Variable & $\begin{array}{l}\text { Killip class } \geq 2 \\
\quad(n=16)\end{array}$ & $\begin{array}{l}\text { Killip class } 1 \\
\quad(n=132)\end{array}$ & $\mathrm{p}$-value \\
\hline \multicolumn{4}{|c|}{ Oxidative stress } \\
\hline Thiol groups $[\mu \mathrm{M}]^{*}$ & $35.6(21.3)$ & $44.1(21.1)$ & 0.046 \\
\hline 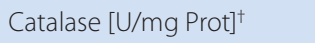 & $102.5 \pm 8.5$ & $93.7 \pm 21.3$ & 0.106 \\
\hline $\mathrm{GR}[\mathrm{U} / \mathrm{mL}]^{\dagger}$ & $8.24 \pm 6.32$ & $7.90 \pm 4.21$ & 0.536 \\
\hline $\mathrm{SOD}[\mathrm{U} / \mathrm{mL}]^{\dagger}$ & $3.0 \pm 0.9$ & $3.1 \pm 1.1$ & 0.705 \\
\hline \multicolumn{4}{|c|}{ Necrosis } \\
\hline Peak CK $[U / L]^{¥}$ & $4228.5(3185.3)$ & $\begin{array}{c}1731.5 \\
(2494.0)\end{array}$ & $<0.001$ \\
\hline \multicolumn{4}{|c|}{ Stretch } \\
\hline BNP $[\mathrm{pg} / \mathrm{mL}]^{¥}$ & $82.4(128.6)$ & $30.0(81.4)$ & $<0.001$ \\
\hline \multicolumn{4}{|c|}{ Inflammation } \\
\hline Sedimentation $[\mathrm{mm} / \mathrm{h}]^{*}$ & $21(32.8)$ & $18.0(18.0)$ & 0.451 \\
\hline C-reactive protein $[\mathrm{mg} / \mathrm{L}]^{*}$ & $30.9(45.2)$ & $9.4(10.5)$ & 0.002 \\
\hline Leukocyte count $\left[\times 10^{9} / \mathrm{L}\right]^{\dagger}$ & $14.7 \pm 4.4$ & $11.1 \pm 3.1$ & $<0.001$ \\
\hline Neutrophil count $\left[\times 10^{9} / \mathrm{L}\right]^{\dagger}$ & $85.8 \pm 3.9$ & $78.3 \pm 9.2$ & $<0.001$ \\
\hline Fibrinogen $[\mathrm{g} / \mathrm{L}]^{*}$ & $2.0(0.0)$ & $2.0(1.0)$ & 0.815 \\
\hline
\end{tabular}

${ }^{\dagger}$ values are mean $\pm \mathrm{SD} ;{ }^{*}$ values are median $(\mathrm{IQR}) ; \mathrm{GR}$ - glutathione reductase; SOD - superoxide dismutase; CK - creatine kinase; BNP - B-type natriuretic peptide.

${ }^{\dagger}$ values are mean $\pm S D ;{ }^{\ddagger}$ values are median (interquartile range $-I Q R$ ) $\mathrm{BMI}$ - body mass index; eGFR - estimated glomerular filtration rate; LVESD - left ventricular end-systolic diameter; LVEDD - left ventricular end-diastolic diameter; LA - left atrium; WMSI - wall motion score index; STR - resolution of ST-segment elevation; IRA - infarct related artery; LM - left main coronary artery; LAD - left anterior descending artery; LCX - left circumflex artery; RCA - right coronary artery; TIMI - thrombolysis after myicardial infarction; ACE - angiotensin-converting enzyme. 


\section{Independent predictors of left ventricular systolic dysfunction and acute heart failure}

After including all significant variables from the univariate logistic regression into multivariate logistic regression model, 4 variables remained significant predictors of LVEF $\leq 40 \%$ : thiol groups (OR 0.82, 95\% CI 0.698-0.966; $\mathrm{p}=0.017$ ), peak CK (OR 1.001, 95\% CI 1.000-1.001; $\mathrm{p}=0.003)$, anterior wall infarction (OR 22.21, 95\% CI 6.81-72.48; p < 0.001), and age (OR 1.06, 95\% CI 1.01-1.11; p = 0.021) (Table 4).

Table 5 shows the results of multiple logistic regression analysis employed to identify independent predictors of $\mathrm{HF}$ during hospitalization (Killip $\geq 2$ ). Out of 16 variables significantly associated with the Killip $\geq 2$ in univariate logistic regression analysis, 5 appeared to be independently associated with acute $\mathrm{HF}$ in multivariable analysis: catalase (OR 1.11,95\% CI 1.02-1.21; $\mathrm{p}=0.020$ ), BNP (OR 1.01, 95\% CI 1.001-1.012; $\mathrm{p}=0.028)$, leucocytes (OR 1.32, 95\% CI 1.04-1.67; $\mathrm{p}=0.025)$, neutrophil count (OR 1.20, 95\% CI 1.04-1.39; $\mathrm{p}=0.014)$, and size of LA (OR 5.76, 95\% CI 1.31-25.37; p = 0.021).

Table 4. Independent predictors of left ventricular systolic dysfunction $(\mathrm{LVEF} \leq 40 \%)$

\begin{tabular}{|l|c|c|c|}
\multicolumn{1}{|c|}{ Variable* } & OR & $95 \% \mathrm{Cl}$ & $\mathrm{p}$-value \\
\hline Thiol groups & 0.821 & $0.698-0.966$ & 0.017 \\
\hline Peak CK & 1.001 & $1.000-1.001$ & 0.003 \\
\hline Anterior wall infarction & 22.212 & $6.807-72.475$ & $<0.001$ \\
\hline Age & 1.056 & $1.008-1.106$ & 0.021 \\
\hline
\end{tabular}

* Multivariate logistic model was started with 16 variables. The following variables were excluded from the model: BNP, leukocyte count, neutrophil count, C-reactive protein, fibrinogen, STR, LA, RCA, ventricular arrhythmias, LVEDD, male gender and BMI. Odds ratios correspond to 1 unit increase in predictor variable.

CK - creatine kinase; BNP - B-type natriuretic peptide; STR - resolution of ST-segment elevation; LA - left atrium; RCA - right coronary artery; LVEDD - left ventricular end-diastolic diameter; BMI - body mass index.

Table 5. Independent predictors of acute heart failure (Killip class $\geq 2$ )

\begin{tabular}{|l|c|c|c|}
\multicolumn{1}{|c|}{ Variable* } & OR & $95 \% \mathrm{Cl}$ & $\mathrm{p}$-value \\
\hline Catalase & 1.110 & $1.017-1.211$ & 0.020 \\
\hline BNP & 1.006 & $1.001-1.012$ & 0.028 \\
\hline LA & 5.763 & $1.309-25.371$ & 0.021 \\
\hline Leukocyte count & 1.315 & $1.036-1.669$ & 0.025 \\
\hline Neutrophil count & 1.199 & $1.037-1.386$ & 0.014 \\
\hline
\end{tabular}

* Multivariate logistic model was started with 16 variables. The following variables were excluded from the model: thiol groups, CK, C-reactive protein, STR, anterior wall infarction, ventricular arrhythmias, WMSI $\geq 1.5$, LVESD, LVEDD, male gender, and BMI. Odds ratios correspond to 1 unit increase in predictor variable.

BNP - B-type natriuretic peptide; LA - left atrium; CK - creatine kinase; STR - resolution of ST-segment elevation; WMSI - wall motion score index; LVEDD - left ventricular end-diastolic diameter; LVESD - left ventricular end-systolic diameter; BMI - body mass index.

\section{Discussion}

Appearance of left ventricle dysfunction after AMI has a great adverse impact on patient outcomes. Although several biomarkers of HF are well studied and widely accepted as reliable in routine clinical practice, their significance in prediction of LVSD early after AMI remains uncertain. ${ }^{19}$ In this study, we assessed the impact of different biomarkers for developing LVSD and acute HF during hospitalization measured in the early phase of acute myocardial infarction including: markers of necrosis (peak CK), markers of myocardial stretch (BNP), inflammatory markers (CRP, leucocyte and neutrophil count), as well as oxidative stress markers (total thiol groups, catalase, SOD and GR). Peak CK, anterior wall infarction, age, and total thiol groups appeared as independent predictors of LVSD. Interestingly, BNP, although being a sensitive marker of HF, was not an independent predictor for future development of LVEF $\leq 40 \%$. However, BNP levels were significantly higher in patients with systolic dysfunction.

Peak CK correlated with the extent of myocardial necrosis, a major factor contributing to the development of LVSD and HF after admission. ${ }^{11}$ Similarly, Hamdan et al. found that peak CK in patients after STEMI undergoing primary PCI was one of the most powerful predictors of LV function. ${ }^{1}$ Anterior localization of myocardial infarction is generally associated with extensive myocardial necrosis and worse prognosis. ${ }^{4}$ Age as a predictor of adverse outcomes after STEMI is also well known and described in many studies. ${ }^{20}$

Thiol groups showed a direct correlation with EF and were an independent predictor for LVSD in our study group. To the best of our knowledge, the predictive role of thiol groups for LVSD development after AMI has not been described, albeit a decrease of serum thiol group was observed in coronary artery disease patients and AMI. ${ }^{8,21}$ Moreover, the disulphide/total thiol ratio was independently related to AMI. ${ }^{8}$ Different thiol compounds interact with circulating nitric oxide and produce S-nitrosothiols, a potent endogenous vasodilators. ${ }^{22}$ Protein S-nitrosylation has an essential role in inflammatory response, apoptosis, nitric oxide synthase activity, myocardial contractility, and response to hypoxia and may be involved in pathogenesis of atherosclerotic heart disease, pulmonary hypertension and cardiac arrhythmias. ${ }^{23}$ Considering that detection of total thiol groups is a cheap, simple one-step colorimetric method, that can easily be automated, their determination may be in the future of great importance in AMI.

Not all patients with LVSD develop acute HF, and not all patients who develop HF have LVSD. In our study, independent predictors of acute HF (Killip $\geq 2$ ) were BNP, leucocytes, neutrophils, catalase, and LA size. Although patients with acute HF had lower concentrations of thiol groups, they were not an independent predictor of HF, while they were an independent predictor of LVSD probably because of the relatively small number of patients who developed HF and skewed distribution of thiol groups (box 
plot shown in supplementary material). The evaluation of BNP concentration is a useful tool for detecting LVSD and a prognostic factor for an adverse clinical outcome after STEMI. BNP correlates with myocardial damage, LVSD and acute HF. ${ }^{24,25}$ Similarly, in our patients, BNP levels were significantly higher when LVSD was present, and displayed a significant negative correlation with LVEF. Interestingly, some authors found that the levels of NT-proBNP early after AMI do not predict the development of LVSD. ${ }^{26}$ Higher BNP levels in patients with LVSD were not an independent predictor of low EF. However, BNP was a predictor of acute HF during hospitalization. This could be explained by the fact that BNP and NT-proBNP are stretch dependent peptide derivatives whose synthesis requires some time to complete. ${ }^{19}$

Leucocytes and neutrophils were also independent predictors of acute HF in our group of patients. Neutrophil and leucocyte counts were described as predictors of severe microvascular injury and LVSD. ${ }^{27}$ Neutrophils release variety of inflammatory cytokines that are directly involved in endothelial injury. After cholesterol crystals ingestions, neutrophils produce neutrophil extracellular traps (NET), structures composed of extracellular neutrophil DNA and proteins that are highly thrombogenic. ${ }^{28}$ Moreover, NETs induce macrophages to produce IL-1 $\beta$, a major cytokine responsible for endothelial inflammation. In animal models NETs formed in situ after reperfusion are responsible for myocardial microvasculature obstruction and consequent appearance of HF. ${ }^{29}$

Catalase has an important role in hydrogen peroxide degradation. Despite its relatively low toxicity, hydrogen peroxide may mediate synthesis of highly reactive hydroxyl radical. Patients with coronary artery disease have lower catalase levels and increased oxidative stress than aged matched controls. ${ }^{30}$ Data related to catalase status in AMI is rather conflicting. Several papers reported lower as well as higher catalase activity in AMI in comparison to healthy controls. ${ }^{9}$ In the end-stage, failing heart catalase gene as well as catalase tissue activity were clearly elevated, probably as compensatory mechanism in response to pronounced oxidative stress. ${ }^{10}$ In our group of patients, catalase at admission was an independent predictor for development of acute HF.

\section{Conclusions}

In this study, we have shown for the first time that thiol groups and catalase are independent predictors of AMI complications - LVSD and acute HF, respectively. Apart from the routinely used biomarkers of necrosis and myocardial stretch, thiol groups and catalase may provide additional information regarding the risk for LVSD development. Considering the simplicity of laboratory procedures necessary to determine catalase and thiol group and their usefulness, these 2 biomarkers could be important and novel tools for risk stratification in AMI.

\section{References}

1. Hamdan A, Kornowski R, Solodky A, Fuchs S, Battler A, Assali AR. Predictors of left ventricular dysfunction in patients with first acute anterior myocardial infarction undergoing primary angioplasty. Isr Med Assoc J. 2006;8:532-535.

2. Świątkiewicz I, Magielski P, Woźnicki M, et al. Occurrence and predictors of left ventricular systolic dysfunction at hospital discharge and in long-term follow-up after acute myocardial infarction treated with primary percutaneous coronary intervention. Kardiol Pol. 2012;70:329-340.

3. Frangogiannis NG, Youker KA, Rossen RD, et al. Cytokines and the microcirculation in ischemia and reperfusion. J Mol Cell Cardiol. 1998; 30:2567-2576.

4. McClements BM, Weyman AE, Newell JB, Picard MH. Echocardiographic determinants of left ventricular ejection fraction after acute myocardial infarction. Am Heart J. 2000;140:284-290.

5. McMurray JJ, Adamopoulos S, Anker SD, et al. ESC Guidelines for the diagnosis and treatment of acute and chronic heart failure 2012: The Task Force for the Diagnosis and Treatment of Acute and Chronic Heart Failure 2012 of the European Society of Cardiology. Developed in collaboration with the Heart. Eur Heart J. 2012;33:1787-1847.

6. Bayes-Genis A, Ordonez-Llanos J. Multiple biomarker strategies for risk stratification in heart failure. Clin Chim Acta. 2015;443:120-125.

7. De Boer RA, Daniels LB, Maisel AS, Januzzi JL. State of the art: Newer biomarkers in heart failure. Eur J Heart Fail. 2015;17:559-569.

8. Kundi $\mathrm{H}$, Ates I, Kiziltunc $\mathrm{E}$, et al. A novel oxidative stress marker in acute myocardial infarction; Thiol/disulphide homeostasis. Am J Emerg Med. 2015;33:1567-1571.

9. Bagatini MD, Martins CC, Battisti V, et al. Oxidative stress versus antioxidant defenses in patients with acute myocardial infarction. Heart Vessels. 2011;26:55-63.

10. Dieterich S, Bieligk U, Beulich K, Hasenfuss G, Prestle J. Gene expression of antioxidative enzymes in the human heart: Increased expression of catalase in the end-stage failing heart. Circulation. 2000;101: 33-39.

11. Steg PG, James SK, Atar D, et al. ESC Guidelines for the management of acute myocardial infarction in patients presenting with ST-segment elevation. Eur Heart J. 2012;33:2569-2619.

12. Matetzky S, Novikov M, Gruberg L, et al. The significance of persistent ST elevation versus early resolution of ST segment elevation after primary PTCA. J Am Coll Cardiol. 1999;34:1932-1938.

13. Sheehan FH, Braunwald E, Canner P, et al. The effect of intravenous thrombolytic therapy on left ventricular function: A report on tissue-type plasminogen activator and streptokinase from the Thrombolysis in Myocardial Infarction (TIMI Phase I) trial. Circulation. 1987;75:817-829.

14. Lang RM, Badano LP, Mor-Avi V, et al. Recommendations for Cardiac Chamber Quantification by Echocardiography in Adults: An Update from the American Society of Echocardiography and the European Association of Cardiovascular Imaging. J Am Soc Echocardiogr. 2015;28:1-39.

15. Sedlak J, Lindsay RH. Estimation of total, protein-bound, and nonprotein sulfhydryl groups in tissue with Ellman's reagent. Anal Biochem. 1968;25:192-205.

16. Góth L. A simple method for determination of serum catalase activity and revision of reference range. Clin Chim Acta. 1991;196:143-151.

17. Sun M, Zigman S. An improved spectrophotometric assay for superoxide dismutase based on epinephrine autoxidation. Anal Biochem. 1978;90:81-89.

18. Hosmer DW, Hjort NL. Goodness-of-fit processes for logistic regression: Simulation results. Stat Med. 2002;21:2723-2738.

19. Gaggin HK, Januzzi JL. Biomarkers and diagnostics in heart failure. Biochim Biophys Acta. 2013;1832:2442-2450.

20. Bhave PD, Hoffmayer KS, Armstrong EJ, et al. Predictors of depressed left ventricular function in patients presenting with ST-elevation myocardial infarction. Am J Cardiol. 2012;109:327-331.

21. Kadota K, Yui Y, Hattori R, Murohara Y, Kawai C. Decreased sulfhydryl groups of serum albumin in coronary artery disease. Jpn Circ J. 1991;55:937-941.

22. Liu T, Schroeder HJ, Wilson SM, et al. Local and systemic vasodilatory effects of low molecular weight S-nitrosothiols. Free Radic Biol Med. 2016;91:215-223. 
23. Maron BA, Tang SS, Loscalzo J. S-nitrosothiols and the S-nitrosoproteome of the cardiovascular system. Antioxid Redox Signal. 2013;18: 270-287.

24. Richards AM. B-type natriuretic peptides and ejection fraction for prognosis after myocardial infarction. Circulation. 2003;107:2786-2792.

25. Talwar S, Squire IB, Downie PF, et al. Profile of plasma N-terminal proBNP following acute myocardial infarction; Correlation with left ventricular systolic dysfunction. Eur Heart J. 2000;21:1514-1521.

26. Ben-Dor I, Haim M, Rechavia E, et al. Serum NT-proBNP concentrations in the early phase do not predict the severity of systolic or diastolic left ventricular dysfunction among patients with ST-elevation acute myocardial infarction. Angiology. 2007;57:686-693.

27. Takahashi T, Hiasa Y, Ohara Y, et al. Relation between neutrophil counts on admission, microvascular injury, and left ventricular func- tional recovery in patients with an anterior wall first acute myocardial infarction treated with primary coronary angioplasty. Am J Cardiol. 2007;100:35-40.

28. Warnatsch A, loannou M, Wang Q, Papayannopoulos V. Inflammation. Neutrophil extracellular traps license macrophages for cytokine production in atherosclerosis. Science. 2015;349:316-320.

29. Ge L, Zhou X, Ji WJ, et al. Neutrophil extracellular traps in ischemiareperfusion injury-induced myocardial no-reflow: Therapeutic potential of DNase-based reperfusion strategy. Am J Physiol Heart Circ Physiol. 2015;308:500-509.

30. Pytel E, Olszewska-Banaszczyk M, Koter-Michalak M, Broncel M. Increased oxidative stress and decreased membrane fluidity in erythrocytes of CAD patients. Biochem Cell Biol. 2013;91:315-318. 